\title{
Correlação entre as classificações de Pfirrmann e Modic na degeneração do disco intervertebral lombar
}

\author{
Correlation between Pfirrmann and Modic classifications in the \\ degeneration of lumbar intervertebral disc \\ Correspondencia entre las clasificaciones de Pfirrmann y Modic en la \\ degeneración del disco intervertebral lumbar
}

\author{
Marcio Squassoni Leite' \\ Rafael Paiva Luciano' \\ Délio Eulálio Martins ${ }^{2}$ \\ Marcelo Wajchenberg ${ }^{3}$ \\ Eduardo Barros Puertas ${ }^{4}$
}

\section{RESUMO}

Objetivo: correlacionar as alterações encontradas nas ressonâncias magnéticas lombares quanto às classificações de Pfirrmann e Modic. Métodos: foram selecionadas 54 ressonâncias lombossacras de pacientes ambulatoriais (23 homens e 31 mulheres) que já se encontravam em investigação por sua comorbidade. Foram classificados 264 discos intervertebrais (L1 a S1) quanto ao grau de degeneração segundo Pfirrmann. A presença de alterações de sinal de corpo vertebral foi registrada quanto à classificação de Modic. Foi aplicado teste $\chi^{2}$, adotando níveis de significância inferiores a $0,05(\mathrm{a}=5 \%)$. Resultados: a média de idade pesquisada foi de 48,4 anos (26 a 77 anos). Observou-se maior prevalência de Pfirrmann tipo IV (31,1\%). Em 88,3\% da amostra o sinal de Modic estava ausente, assim como Modic 3; 60\% das alterações Modic 2 foram relacionadas ao Pfirrmann tipo V, e $36,4 \%$ das

\section{ABSTRACT}

Objective: to correlate the changes found in the lumbar magnetic resonance imaging according to Modic and Pfirrmann classifications. Methods: lumbar spine resonances of 54 outpatients were selected (23 men and 31 women), who were already under investigation because of their comorbidity. According to their degree of Pfirrmann classification, 264 intervertebral discs (L1 to S1) were classified. The presence of signal abnormalities of the vertebral body was recorded as Modic classification. The $\chi^{2}$ was applied, adopting significance levels below 0.05 ( $a=5 \%)$. Results: The mean age studied was of 48.4 years (26-77 years). A higher prevalence of Pfirrmann type IV (31.1\%) was observed. In $88.3 \%$ of the sample, Modic was absent, and also Modic 3; $60 \%$ of Modic 2 was related to Pfirrmann type V, and $36.4 \%$ of Modic
\end{abstract}

\section{RESUMEN}

Objetivo: correlacionar los cambios observados en las resonancias magnéticas lumbares con relación a las clasificaciones de Pfirrmann y Modic. Métodos: resonancias lumbar sacra fueron seleccionadas de 54 pacientes de ambulatorios (23 hombres y 31 mujeres), que ya estaban siendo investigados por su comorbilidad. Doscientos sesenta y cuatro discos intervertebrales (L1 a S1) se clasificaron según su grado de clasificación de Pfirrmann. La presencia de anomalías en la señal del cuerpo vertebral se registró como la clasificación de Modic. Se aplicó el teste $\chi^{2}$, adoptando niveles de significancia por debajo de 0,05 ( $a=5 \%)$. Resultados: la media de edad estudiada fue 48,4 años (26-77 años). Se observó una mayor prevalencia de Pfirrmann tipo IV (el 31,1\%). En 88,3\% de la muestra, el señal Modic estaba ausente, así como Modic 3. Sesenta por ciento de Mo-

\footnotetext{
Departamento de Ortopedia e Traumatologia da Escola Paulista de Medicina da Universidade Federal de São Paulo - UNIFESP - São Paulo (SP), Brasil.

'Médico Residente (R5) do Grupo da Coluna da Disciplina de Ortopedia do Departamento de Ortopedia e Traumatologia da Escola Paulista de Medicina da Universidade Federal de São Paulo - UNIFESP - São Paulo (SP), Brasil.

${ }^{2}$ Mestre; Médico Assistente do Grupo da Coluna da Disciplina de Ortopedia do Departamento de Ortopedia e Traumatologia em Ortopedia e Traumatologia da Escola Paulista de Medicina da Universidade Federal de São Paulo - UNIFESP - São Paulo (SP), Brasil.

${ }^{3}$ Doutor; Médico Assistente do Grupo da Coluna da Disciplina de Ortopedia do Departamento de Ortopedia e Traumatologia da Escola Paulista de Medicina da Universidade Federal de São Paulo - UNIFESP - São Paulo (SP), Brasil.

${ }^{4}$ Livre-docente; Professor Associado da Disciplina de Ortopedia do Departamento de Ortopedia e Traumatologia da Escola Paulista de Medicina da Universidade Federal de São Paulo - UNIFESP - São Paulo (SP), Brasil.
} 
alterações Modic 1 foram igualmente distribuídas entre Pfirrmann tipo IV e V. Encontrou-se associação estatisticamente significante entre as alterações de Modic e de Pfirrmann $(\mathrm{p}<0,001)$. Conclusão: houve uma clara associação entre as classificações estudadas ao se comparar os estágios mais avançados de Pfirrmann (IV e V) com Modic tipo 1 e 2 .
1 was equally distributed among Pfirrmann type IV and V. Statistically significant association between Modic changes and Pfirrmann $(p<0.001)$ was found. Conclusion: there was a clear association between the classifications studied, comparing the stages of Pfirrmann (IV and V) with Modic type 1 and 2.

KEYWORDS: Spine; Intervertebral disk; Intervertebral disk degeneration; Magnetic resonance imaging; Low back pain dic 2 estaban relacionados con Pfirrmann tipo $V$, y el 36,4\% de las alteraciones Modic 1 fueron por igual entre Pfirrmann tipo IV y V. Se encontró una asociación estadisticamente significativa entre los cambios de Modic y Pfirrmann $(p<0,001)$. Conclusión: existe una clara asociación entre las clasificaciones estudiadas, en comparación de las últimas etapas de Pfirrmann (IV y V) con Modic tipos 1 y 2.
DESCRITORES: Coluna vertebral; Disco intervertebral; Degeneração do disco intervertebral; Imagem por ressonância magnética; Dor lombar
DESCRIPTORES: Columna vertebral; Disco intervertebral; Degeneración del disco intervertebral; Imagen por resonancia magnética; Dolor de la región lumbar

\section{INTRODUÇÃO}

A doença degenerativa do disco é considerada um dos principais fatores de dor lombar baixa ${ }^{1-4}$. Um achado frequente sobre essa condição, visto em ressonância magnética e conhecido como alteração tipo Modic, é creditada por alguns autores também como fonte de dor $^{5-9}$, principalmente no estágio Modic I, que corresponde ao processo inflamatório inicial. Estudos clínicos e experimentais relacionam dores lombares crônicas com doenças dos discos intervertebrais em até $40 \%{ }^{2}$, gerando incapacidades funcionais e laborais, com impacto socioeconômico de grande porte ${ }^{3,4}$.

Vários fatores são determinantes da complexa fisiopatologia da degeneração discal, que ainda é pouco compreendida ${ }^{3,10,11}$. O disco intervertebral normal apresenta anatomia e bioquímica que permitem absorver e dissipar cargas transmitidas entre os segmentos da coluna ${ }^{2,3}$, sendo formado por três estruturas: o ânulo fibroso, o núcleo pulposo e a placa terminal. Por volta da terceira década da vida, com a desidratação do núcleo pulposo e a modificação das estruturas moleculares de seus componentes, inicia-se o processo de degeneração discal ${ }^{12,13}$.

Os sinais tardios encontrados nesse processo são: a) perda de altura do disco nas radiografias laterais da coluna; b) formação de osteófitos; c) nódulo de Schmorl; d) esclerose da placa terminal; e) sinal do vácuo ${ }^{14}$. Para esses achados radiográficos, conjuntamente, utiliza-se a denominação osteocondrose intervertebral ${ }^{3,15}$. No exame de ressonância magnética são descritos: diminuição de altura, abaulamentos, herniações, presença de alterações da placa terminal tipo Modic e do disco intervertebral, como apresentado pela classificação de Pfirrmann ${ }^{3,15-17}$.

Embora grandes perdas de altura discal impliquem extensa desestruturação do mesmo, não há uma correlação direta bem estabelecida entre o início da degeneração vista em ressonância (classificação de Pfirrmann ${ }^{16}$ ) com alteração de sinal do corpo vertebral (classificação de Modic ${ }^{3,15,18-21}$.

Dessa forma, o presente estudo tem como objetivo comparar as alterações degenerativas encontradas na ressonância magnética, segundo a classificação de Modic e Pfirrmann.

\section{MÉTODOS}

Foram analisadas ressonâncias magnéticas da coluna lombossacra (L1 a S1) de 54 pessoas (23 homens e 31 mulheres) que já se encontravam em acompanhamento ambulatorial e em investigação de sua comorbidade, segundo os critérios de inclusão: idade mínima de 18 anos; história de dor lombar aguda ou crônica; que tenham concordado e assinado o Termo de Consentimento Livre e Esclarecido, conforme aprovação do Comitê de Ética em Pesquisa, número 1479/09. Não foram incluídos pacientes que apresentavam as condições: tratamento cirúrgico prévio na coluna vertebral; alterações congênitas radiográficas, como malformações vertebrais; presença de escoliose acima de $15^{\circ} \mathrm{Cobb}$; presença de espondilolistese maior que $20 \%$ de escorregamento; fraturas prévias; doenças tumorais.

Foram classificados 264 discos lombares segundo Pfirrmann, em relação à degeneração do disco intervertebral (Tabela 1 e Figura 1).

Alterações do tipo Modic (Figura 2) foram classificadas em três tipos, de acordo com a intensidade do sinal nas ressonâncias magnéticas ponderadas em T1 e T2: tipo 1 - hiposinal em T1 e hipersinal em T2; tipo 2 - hipersinal em T1 e iso ou hipersinal em T2; tipo 3 - hiposinal em T1 e T2 $2^{3,22}$. Quando não se observaram alterações, 


\section{TABELA 1 - Classificação da degeneração discal segundo Pfirrmann}

\begin{tabular}{|c|c|c|c|c|}
\hline Grau & Estrutura & $\begin{array}{l}\text { Distinção entre o } \\
\text { núcleo e ânulo }\end{array}$ & Intensidade do sinal & $\begin{array}{l}\text { Altura disco } \\
\text { intervertebral }\end{array}$ \\
\hline | & Homogêneo, branco brilhante & Clara & $\begin{array}{c}\text { Hipersinal, isointenso com o } \\
\text { líquor }\end{array}$ & Normal \\
\hline$\|$ & $\begin{array}{c}\text { Não homogêneo, com ou sem bandas } \\
\text { horizontais }\end{array}$ & Clara & $\begin{array}{c}\text { Hipersinal, isointenso com o } \\
\text { líquor }\end{array}$ & Normal \\
\hline III & Não homogêneo, cinza & Não clara & Intermediário & $\begin{array}{l}\text { Normal à discreta } \\
\text { diminuição }\end{array}$ \\
\hline IV & Não homogêneo, cinza para preto & Perdida & Intermediário à hiposinal & $\begin{array}{l}\text { Normal à moderada } \\
\text { diminuição }\end{array}$ \\
\hline V & Não homogêneo, preto & Perdida & Hiposinal & $\begin{array}{l}\text { Espaço discal } \\
\text { colapsado }\end{array}$ \\
\hline
\end{tabular}

Traduzido de Pfirrmann et al. ${ }^{16}$.

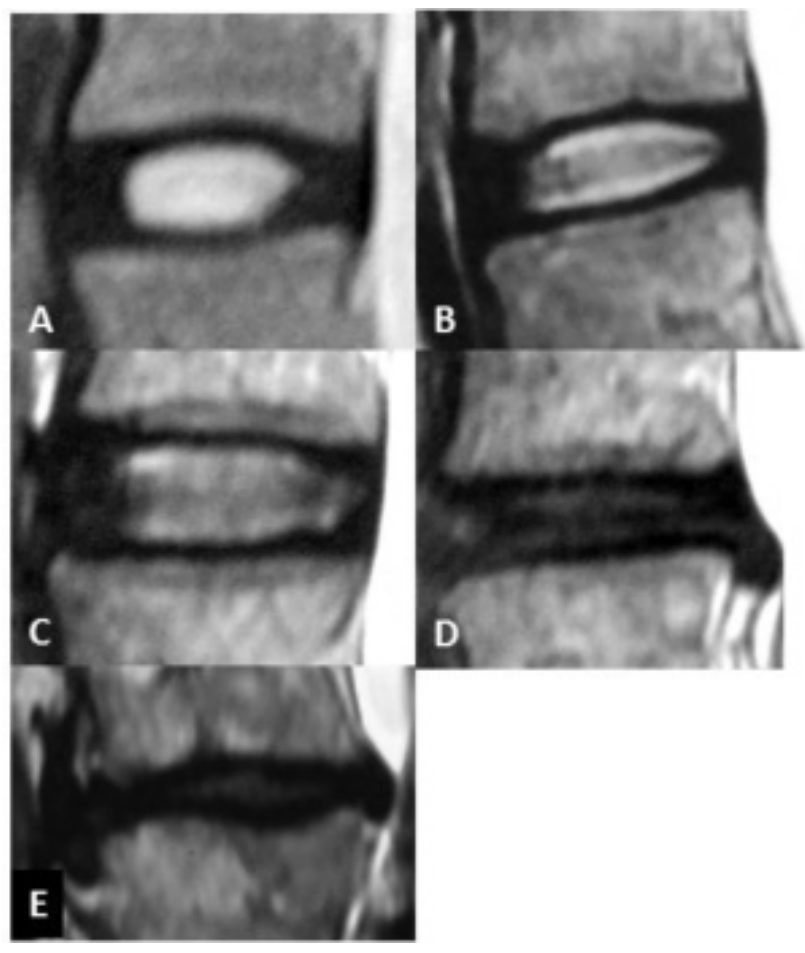

Figura 1

Classificação da degeneração discal lombar, segundo Pfirmann, correspondentes aos graus de I a V, conforme Tabela 1.

para fins estatísticos, essa situação foi classificada como ausente (zero).

Para análise de associação entre os graus Modic e Pfirrmann foi utilizado o teste $\chi^{2}$, da razão de verossimilhança. O nível de significância de $0,05(\alpha=5 \%)$ e os níveis descritivos $(p)$ inferiores a esse valor foram considerados significativos e representados por *. Todas as análises foram feitas pelo software SPSS for Windows versão 15.0.

\section{RESULTADOS}

Foram analisados 54 pacientes, 31 do gênero feminino $(57,4 \%)$ e 23 do gênero masculino $(42,6 \%)$, com média de idade de 48,4 anos, mínino de 26 e máximo de 77 anos.
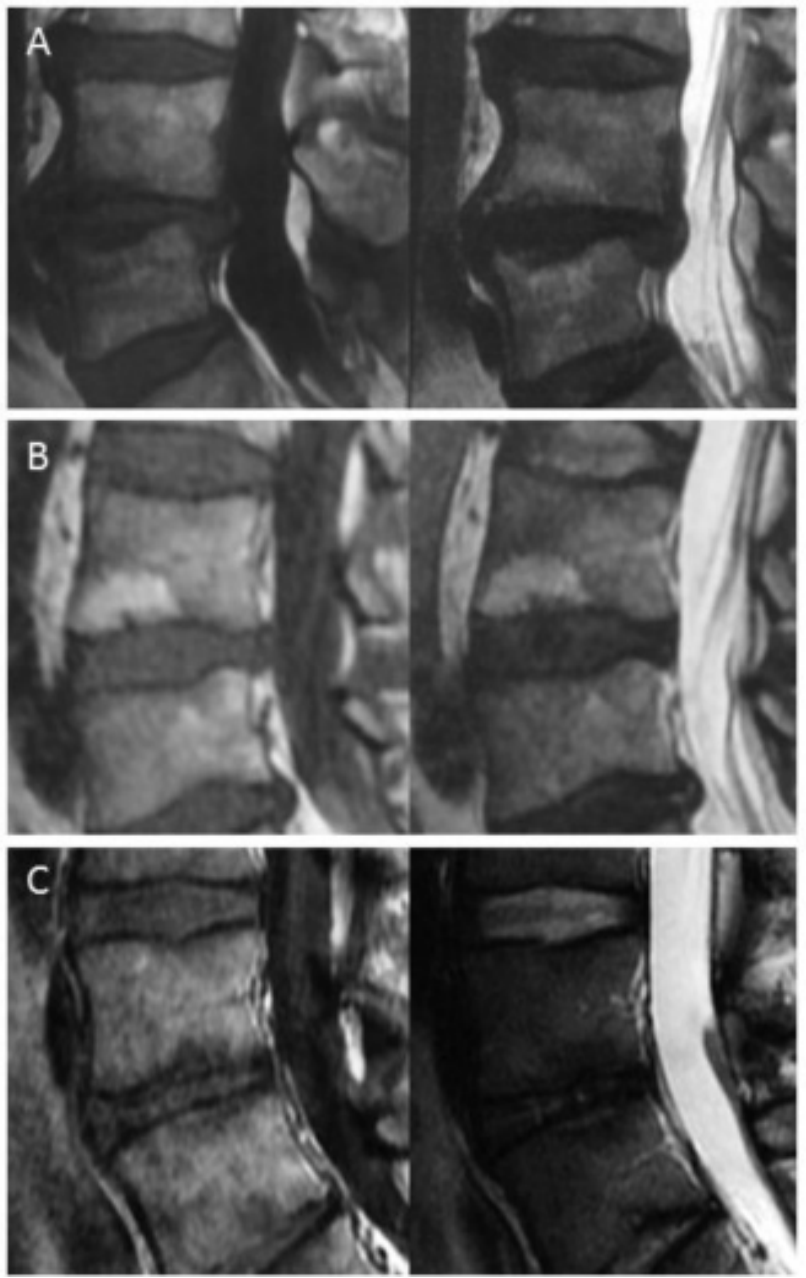

Figura 2

Alterações de corpo vertebral segundo Modic, em ressonância magnética ponderada em T1 e T2. (A) tipo 1; (B) tipo 2; (C) tipo 3.

Nesses pacientes, 264 discos foram classificados quanto a Pfirrmann e quanto à presença de alterações tipo Modic. Em 233 discos (88,3\%) não foi encontrado sinal tipo Modic, e 85,3\% de todos os discos se situavam entre Pfirrmann II e IV. Modic tipo 3 também não foi observado. A 
distribuição para a classificação de Modic e Pfirrmann é apresentada nas Tabelas 2, 3 e 4.

Na Tabela 3, evidenciamos que $100 \%$ dos discos localizados no nível L1/2 não apresentaram sinal de Modic, assim como nos três discos localizados entre VT/S1 (VT: vértebra de transição). Modic tipo 1 e 2 foi mais frequente entre os níveis L4/5 e L5/VT.

TABELA 2 - Características da amostra dos discos em relação ao Pfirrmann e Modic

\begin{tabular}{lc}
\hline Variáveis & $(\mathbf{n}=\mathbf{2 6 4 )}$ \\
\hline Degeneração medular óssea: Modic $-\mathrm{n}(\%)$ & \\
Ausente & $233(88,3)$ \\
Tipo I & $11(4,2)$ \\
Tipo 2 & $20(7,6)$ \\
Degeneração discal: Pfirrmann - n (\%) & \\
Tipo I & $14(5,3)$ \\
Tipo II & $68(25,8)$ \\
Tipo III & $75(28,4)$ \\
Tipo IV & $82(31,1)$ \\
Tipo V & $25(9,5)$ \\
\hline
\end{tabular}

Com relação a classificação de Pfirrmann, não foi encontrado o tipo I entre os níveis L5/VT e VT/S1. Do total de 54 discos localizados em L4/5, 24 (44,4\%) se apresentaram como tipo IV, e em $66,7 \%$ dos três discos encontrados em L5/VT. O tipo V foi mais frequente no nível L5/ S1 com 15 discos $(29,4 \%)$, dos 51 observados nesse nível (Tabela 4).

No grupo de discos com Modic "ausente" houve equilíbrio entre Pfirrmann tipo II, tipo III e tipo IV, concentrando $90,2 \%$ dos discos. Já no grupo de discos com Modic tipo 1, houve equilíbrio entre Pfirrmann tipo IV e tipo V, totalizando $72,8 \%$ dos discos. Por fim, no grupo de discos com Modic tipo 2, houve predomínio de Pfirrmann tipo $\mathrm{V}$ com $60 \%$ dos discos. A situação Modic tipo 3 não foi encontrada dentro da amostra estudada.

Após análise dos dados, foi encontrada associação estatisticamente significativa entre as alterações de Modic e Pfirrmann $(\mathrm{p}<0,001)$. Das alterações Modic 2, 60\% foram relacionadas ao Pfirrmann tipo V, e 36,4\% das alterações Modic 1 foram igualmente distribuídas entre Pfirrmann tipo IV e V (Tabela 5).

TABELA 3 - Distribuição de frequências do grau de degeneração medular óssea (Modic) dos discos segundo o nível

\begin{tabular}{lccccccc}
\hline Degeneração & \multicolumn{7}{c}{ Nível (disco intervertebral) } \\
\cline { 2 - 7 } medularóssea (Modic) & L1/2 n (\%) & L2/3 n (\%) & L3/4 n (\%) & L4/5 n (\%) & L5/S1 n (\%) & L5/VT n (\%) & VT/S1 n (\%) \\
\hline Ausente & $45(100,0)$ & $53(98,1)$ & $51(94,4)$ & $41(75,9)$ & $39(76,5)$ & $1(33,3)$ & $3(100,0)$ \\
Tipo 1 & $0(0,0)$ & $1(1,9)$ & $0(0,0)$ & $5(9,3)$ & $4(7,8)$ & $1(33,3)$ & $0(0,0)$ \\
Tipo 2 & $0(0,0)$ & $0(0,0)$ & $3(5,6)$ & $8(14,8)$ & $8(15,7)$ & $1(33,3)$ & $0(0,0)$ \\
Total & 45 & 54 & 54 & 54 & 51 & 3 & 3 \\
\hline
\end{tabular}

VT: Vértebra de transição.

TABELA 4 - Distribuição de frequências do grau de degeneração discal (Pfirrmann) dos discos segundo o nível

\begin{tabular}{|c|c|c|c|c|c|c|c|}
\hline \multirow{2}{*}{$\begin{array}{l}\text { Degeneração discal } \\
\text { Pfirrmann }\end{array}$} & \multicolumn{7}{|c|}{ Nível (disco intervertebral) } \\
\hline & L1/2 n (\%) & L2/3 n (\%) & L3/4 n (\%) & L4/5 n (\%) & L5/S1 n (\%) & L5/VT n (\%) & VT/S1 n (\%) \\
\hline Tipo I & $3(6,7)$ & $4(7,4)$ & $4(7,4)$ & $2(3,7)$ & $1(2,0)$ & $0(0,0)$ & $0(0,0)$ \\
\hline Tipo II & $15(33,3)$ & $21(38,9)$ & $18(33,3)$ & $8(14,8)$ & $4(7,8)$ & $0(0,0)$ & $2(66,7)$ \\
\hline Tipo III & $17(37,8)$ & $17(31,5)$ & $15(27,8)$ & $14(25,9)$ & $12(23,5)$ & $0(0,0)$ & $0(0,0)$ \\
\hline Tipo IV & $10(22,2)$ & $11(20,4)$ & $15(27,8)$ & $24(44,4)$ & $19(37,3)$ & $2(66,7)$ & $1(33,3)$ \\
\hline Tipo V & $0(0,0)$ & $1(1,9)$ & $2(3,7)$ & $6(11,1)$ & $15(29,4)$ & $1(33,3)$ & $0(0,0)$ \\
\hline Total & 45 & 54 & 54 & 54 & 51 & 3 & 3 \\
\hline
\end{tabular}

VT: vértebra de transição.

TABELA 5 - Distribuição do grau de degeneração discal (Pfirrmann) segundo o grau de degeneração medular óssea (Modic)

\begin{tabular}{lccc}
\hline \multirow{2}{*}{ Grau de degeneração discal (Pfirrmann) } & \multicolumn{3}{c}{ Grau de degeneração medular óssea (Modic) } \\
\cline { 2 - 4 } & Ausente $(\mathbf{n = 2 3 3 )}$ & Tipo 1 (n=11) & Tipo 2 ( $\mathbf{n = 2 0 )}$ \\
\hline Tipo I & $14(6,0 \%)$ & $0(0,0 \%)$ & $0(0 \%)$ \\
Tipo II & $67(28,8 \%)$ & $1(9,1 \%)$ & $0(0 \%)$ \\
Tipo III & $69(29,6 \%)$ & $2(18,2 \%)$ & $4(20 \%)$ \\
Tipo IV & $74(31,8 \%)$ & $4(36,4 \%)$ & $4(20 \%)$ \\
Tipo V & $9(3,9 \%)$ & $4(36,4 \%)$ & $12(60 \%)$ \\
Comparação & & $p<0,001 *$ & \\
\hline
\end{tabular}




\section{DISCUSSÃO}

A degeneração discal é um problema prevalente, que geralmente se inicia após a segunda década de vida e aumenta com o tempo. Cerca de $40 \%$ das causas de dores lombares crônicas é de origem discogênica ${ }^{2}$. Apesar disso, a etiologia ainda não está bem esclarecida.

Pfirrmann apresentou sua classificação para degeneração discal em $2001^{16}$, demonstrando boa reprodutibilidade inter e intraobservadores, posteriormente confirmada por Puertas et al. ${ }^{23}$ e Zou et al. ${ }^{20}$. Outros autores propuseram modificadores nesta classificação ${ }^{17,24}$ ressaltando que, em pacientes mais velhos, até $87 \%$ dos discos lombares são incluídos entre os tipos III e IV.

Neste estudo foram avaliados pacientes com média de idade de 48,4 anos ( 26 a 77 anos) e total de discos tipo III e IV de 59,5\%. Pfirmann incluiu em seu trabalho pacientes com idade mínima de dez anos, idade em que, sabidamente, o processo degenerativo e as alterações nos exames de ressonância magnética ainda são raramente encontrados ${ }^{16}$. Assim, obteve um percentual menor de discos tipo III e IV $(45 \%)$. Sua maior porcentagem era de discos tipo II (27\%), diferentemente do encontrado nesta amostra, em que o tipo IV foi o de maior porcentagem $(31,1 \%)$, já que a idade mínima se apresentava na terceira década de vida - idade em que se inicia o processo de degeneração $0^{2-4}$.

Modic et al. ${ }^{15}$ apresentaram sua classificação para as alterações de sinal encontradas nos corpos vertebrais em 1988. Desde então, a mesma vem sendo utilizada e mostrando boa reprodutibilidade e praticidade ${ }^{25}$, como apontado em uma recente revisão sistemática ${ }^{26}$.

Alterações do tipo Modic são largamente relacionadas, por diversos autores, como fonte de dor lombar ${ }^{4,7,8,11,15}$, principalmente no tipo 1, como apontado por Albert e Manniche ${ }^{6}$. Porém, da mesma maneira que alterações degenerativas discais permanecem com etiologia desconhecida, as mudanças de sinal dos corpos vertebrais são indicadas por diversas causas, como: a) alterações de arquitetura interna dos discos que liberam mediadores químicos ou que facilitariam microfraturas do platô vertebral $^{27}$; b) hérnia discal ${ }^{27}$; c) causas infecciosas ${ }^{28}$; d) nódulos de Schmorl ${ }^{5}$; e) causas tumorais ${ }^{26}$.

No presente estudo, a grande maioria dos pacientes não apresentava alterações tipo Modic (88,3\%), além de não ser encontrado nenhum Modic tipo 3, situação considerada rara ${ }^{5,15,26}$. Observamos que os níveis L4/L5 e L5/S1 são os mais comuns a apresentarem alterações nas imagens de ressonância magnética, assim como Modic ${ }^{15}$. Além disso, na presença de VT, até 2/3 apresentaram Modic tipos $1 \mathrm{e}$ 2 , porém, devido ao número muito pequeno de VT observados em nossa amostra (Tabela 3), esse dado é apenas observacional.

Com relação à quantidade de discos degenerados relatados neste estudo, e considerando discos normais os que apresentam Pfirmann menor ou igual a II, conforme $\mathrm{Zuo}^{29}$, e discos doentes os maiores do que tipo II, observou-se que $69 \%$ dos discos estavam doentes (Pfirrmann III, IV e $\mathrm{V})$ - valor este maior do que o citado por $\mathrm{Zuo}^{29}(54 \%)$, que utilizou 37 discos de 17 colunas lombares de cadáveres humanos em seu estudo.

Houve uma associação estatística significativa $(p<0,001)$ entre as duas classificações (Tabela 5). Não encontramos na literatura estudos que comparem a presença de Modic com Pfirrmann e que nos permitisse discutir os resultados observados. Apontamos para um possível fator limitante verificado neste estudo: a quantidade de pacientes. Mais pacientes necessitam ser acrescentados ao grupo avaliado para se obter resultados mais consistentes.

\section{CONCLUSÃO}

Houve uma clara associação entre as classificações estudadas ao se comparar os estágios mais avançados de Pfirrmann (IV e V) com Modic tipo 1 e 2.

\section{REFERÊNCIAS}

1. Auerbach JD, Johannessen W, Borthakur A, Wheaton AJ, Dolinskas CA, Balderston RA, et al. In vivo quantification of human lumbar disc degeneration using $\mathrm{T}(1 \mathrm{rho})$-weighted magnetic resonance imaging. Eur Spine J. 2006;15 Suppl 3:S338-44.

2. Biyani A, Haman SP, Andersson GBJ. Lumbar disc desease. In: Rothman-Simeone, The Spine. 5th ed. Philadelphia: Saunders Elsevier; 2006. p. 930-944.
3. Wang C, Auerbach JD, Witschey WR, Balderston RA, Reddy R, Borthakur A. Advances in Magnetic Resonance Imaging for the assessment of degenerative disc disease of the lumbar spine. Semin Spine Surg. 2007;19(2):65-71.

4. Luoma K, Riihimäki H, Luukkonen R, Raininko R, Viikari-Juntura E, Lamminen A. Low back pain in relation to lumbar disc degeneration. Spine (Phila Pa 1976). 2000;25(4):487-92.

5. Zhao JG, Zhang P, Zhang SF, Qi F, Huang WJ, Xia YZ, et al. Modic type III lesions and Schmorl's nodes are the same pathological changes? Med Hypotheses. 2010;74(3):524-6.
6. Albert HB, Manniche C. Modic changes following lumbar disc herniation. Eur Spine J. 2007;16(7):977-82.

7. Jensen TS, Karppinen J, Sorensen JS, Niinimäki J, Leboeuf-Yde C. Vertebral endplate signal changes (Modic change): a systematic literature review of prevalence and association with non-specific low back pain. Eur Spine J. 2008;17(11):1407-22.

8. Albert HB, Kjaer P, Jensen TS, Sorensen JS, Bendix T, Manniche C. Modic changes, possible causes and relation to low back pain. Med Hypotheses. 2008;70(2):361-8. 
9. Hu ZJ, Zhao FD, Fang XQ, Fan SW. Modic changes, possible causes and promotion to lumbar intervertebral disc degeneration. Med Hypotheses. 2009;73(6):930-2.

10. Toyone T, Takahashi K, Kitahara H, Yamagata M, Murakami M, Moriya $\mathrm{H}$. Vertebral bone-marrow changes in degenerative lumbar disc disease. An MRI study of 74 patients with low back pain. J Bone Joint Surg Br. 1994;76(5):757-64.

11. Weishaupt D, Zanetti M, Hodler J, Min K, Fuchs B, Pfirrmann CW, et al. Painful Lumbar Disk Derangement: Relevance of Endplate Abnormalities at MR Imaging. Radiology. 2001;218(2):420-7.

12.Nguyen AM, Johannessen W, Yoder JH, Wheaton AJ, Vresilovic EJ, Borthakur A, et al. Noninvasive quantification of human nucleus pulposus pressure with use of T1rho-weighted magnetic resonance imaging. J Bone Joint Surg Am. 2008;90(4):796-802.

13.Gruber HE, Hanley EN Jr. Analysis of aging and degeneration of the human intervertebral disc. Comparison of surgical specimens with normal controls. Spine (Phila Pa 1976). 1998;23(7):751-7.

14.Madan SS, Rai A, Harley JM. Interobserver error in interpretation of the radiographs for degeneration of the lumbar spine. Iowa Orthop J. 2003;23:51-6.

15.Modic MT, Steinberg PM, Ross JS, Masaryk TJ, Carter JR. Degenerative disk disease: assessment of changes in vertebral body marrow with MR imaging. Radiology. 1988;166(1 Pt 1):193-9.

16.Pfirrmann CW, Metzdorf A, Zanetti M, Hodler J, Boos N. Magnetic resonance classification of lumbar intervertebral disc degeneration. Spine (Phila $\mathrm{Pa}$ 1976). 2001;26(17):1873-8.
17.Puertas EB, Yamashita H, Oliveira VM, Souza PS. Classification of intervertebral disc degeneration by magnetic resonance. Acta Ortop Bras. 2009;17(1):46-9.

18.Boden SD, Davis DO, Dina TS, Patronas NJ, Wiesel SW. Abnormal magnetic-resonance scans of the lumbar spine in asymptomatic subjects. A prospective investigation. J Bone Joint Surg Am. 1990;72(3):4038.

19.Powell MC, Wilson M, Szypryt P, Symonds EM, Worthington BS. Prevalence of lumbar disc degeneration observed by magnetic resonance in symptomless women. Lancet. 1986;2(8520):1366-7.

20.Zou J, Yang H, Miyazaki M, Morishita Y, Wei F, McGovern S, et al. Dynamic bulging of intervertebral discs in the degenerative lumbar spine. Spine (Phila Pa 1976). 2009;34(23):2545-50.

21.Martins DE, Oliveira VM, Alves MT, Wajchenberg M, Landim E, Belloti JC, et al. Correlations between radiographic, magnetic resonance and histological examinations on the degeneration of human lumbar intervertebral discs. Sao Paulo Med J. 2010;128(2):63-8.

22.Jensen MC, Brant-Zawadzki MN, Obuchowski N, Modic MT, Malkasian D, Ross JS. Magnetic resonance imaging of the lumbar spine in people without back pain. N Engl J Med. 1994;331(2):69-73.

23.Puertas EB, Yamashita H, Oliveira VM, Souza PS. Intraobserver and interobserver reproducibility of the Pfirrmann rating of disc degeneration by magnetic resonance imaging. Coluna/Columna. 2008;7(4):330-3.
24. Griffith JF, Wang YJ, Antonio GE, Choi KC, Yu A, Ahuja AT, et al. Modified Pfirrmann grading system for lumbar intervertebral disc degeneration. Spine (Phila Pa 1976). 2007;32(24):E708-12.

25.Jones A, Clarke A, Freeman BJ, Lam KS, Grevitt MP. The Modic classification: inter- and intraobserver error in clinical practice. Spine (Phila Pa 1976). 2005;30(16):1867-9.

26.Zhang YH, Zhao CQ, Jiang LS, Chen XD, Dai LY. Modic changes: a systematic review of the literature. Eur Spine J. 2008;17(10):1289-99.

27.Jensen TS, Kjaer P, Korsholm L, Bendix T, Sorensen JS, Manniche $\mathrm{C}$, et al. Predictors of new vertebral endplate signal (Modic) changes in the general population. Eur Spine J. 2010;19(1):129-35.

28.Albert HB, Manniche C, Sorensen JS, Deleuran BW. Antibiotic treatment in patients with low-back pain associated with Modic changes Type 1 (bone oedema): a pilot study. Br J Sports Med. 2008;42(12):969-73.

29.Zuo J, Saadat E, Romero A, Loo K, Li X, Link TM, et al. Assessment of intervertebral disc degeneration with magnetic resonance single-voxel spectroscopy. Magn Reson Med. 2009;62(5):1140-6.

Correspondência
Marcio Squassoni Leite
Rua Borges Lagoa, 783,5ªndar
CEP: 04038-031 - São Paulo (SP),
Brasil
Tel: 5571-6621
E-mail: squamar79@bol.com.br

